\title{
Toma de decisiones en consumidores de cannabis
}

\section{Decision making in cannabis users}

Jose Ramón Alameda Ballén ${ }^{*}$; Susana Paíno Quesada ${ }^{* *} ;$
Ana Isabel Mogedas Valladares

\author{
* Área de Psicología Básica de la Universidad de Huelva \\ ** Área de Personalidad, Evaluación y Tratamiento Psicológicos de la \\ Universidad de Huelva
}

Enviar correspondencia a:

Jose Ramón Alameda Bailén

Área de Psicología Básica de la Universidad de Huelva

Facultad de Ciencias de la Educación

Avda. de las Fuerzas Armadas, S/N. Campus de El Carmen

21071 Huelva

E-mail: alameda@uhu.es

\section{Resumen}

Diversos estudios neuropsicológicos han demostrado que los consumidores crónicos de cannabis presentan deterioros cognitivos, incluyendo el proceso de toma de decisiones. Por ello, el presente estudio tiene como objetivo evaluar dicho proceso, a través de la hipótesis del marcador somático, en una muestra de 41 consumidores de cannabis comparándolo con un grupo control de igual tamaño, asi como analizar la influencia de la edad, sexo, nivel de estudios, edad de inicio y cantidad de consumo diario. Para ello, se ha utilizado el programa "Cartas" (similar a la lowa Gambling Task), administrando dos versiones: normal e inversa. Los resultados muestran diferencias estadisticamente significativas entre los consumidores de cannabis y el grupo control en la ejecución de la tarea normal e inversa. En el análisis por bloques, el grupo control obtiene puntuaciones superiores en la ejecución de la tarea normal, sin embargo, en la tarea inversa, las diferencias entre ambos grupos se dan en la parte inicial pero no en la final. Ninguna de las variables analizadas (edad, sexo,...) se relaciona significativamente con el rendimiento de la tarea. Estos resultados sugieren la existencia de alteraciones en el proceso de toma de decisiones de los consumidores de cannabis, que pueden relacionarse con la dificultad para generar marcadores somáticos, y no con la insensibilidad a las pérdidas.

Palabras: toma de decisiones, cannabis, lowa Gambling Task, marcador somático. recibido: Diciembre 2011 aceptado: Febrero 2012

\section{Abstract}

Several neuropsychological studies have shown that chronic cannabis users have cognitive impairments, including decision-making process. Therefore, this study aims to evaluate the process, through the somatic marker hypothesis in a sample of 41 cannabis users compared with a control group of equal size, and to analyze the influence of age, sex, education level, age of onset and amount of daily consumption. In order to do that, the software "Cartas" (similar to the lowa Gambling Task), was used, implementing its two versions: normal and reverse. The results show significant differences between cannabis users and control group in the normal and reverse task execution. By block analysis, the control group obtained higher scores in the normal task execution, however, in the reverse task, the differences between groups are present in the initial task execution but not final task execution. None of the analyzed variables (age, sex ...) are significantly related to task performance. These results suggest the existence of alterations in the decision making process of consumers cannabis, which may relate to the difficulty in generating somatic markers, and not for insensitivity punishments insensitivity.

Key words: decision-making, cannabis, lowa Gambling Task, somatic marker. 
E informe del Observatorio Español sobre Drogas -OED-, de la Delegación del Gobierno para el Plan Nacional de Drogas (2009), indica que el cannabis es la sustancia ilegal más consumida en España, al igual que en el resto de Europa (Informe Anual del European Monitoring Centre for Drugs and Drugs Abuse -EMCDDA-, 2010). Los últimos datos del Observatorio Europeo confirman la tendencia a la estabilización o a la baja del consumo, sin embargo sigue siendo muy elevado y su presencia está cobrando un mayor protagonismo en algunos países de Europa oriental. Se estima que en torno a 75,5 millones de europeos adultos de entre 15 y 64 años $(22,5 \%)$ han probado el cannabis en algún momento de su vida, cerca de 23 millones $(6,8 \%)$ lo han consumido durante el último año y 12,5 millones $(12,5 \%)$ lo consumieron en el último mes. España supera a la media europea en relación al consumo, concretamente el 27,3\% de la población afirma haberla probado alguna vez, un 10,1\% lo hizo en el último año y un 7,1\% en el último mes. Este consumo se concentra principalmente en los adultos jóvenes (entre 15 y 34 años), siendo los jóvenes entre 15 y 24 años los que generalmente indican una prevalencia más elevada, con un consumo más extendido entre los hombres (OED, 2009; EMCDDA, 2010).

El consumo de cannabis tiene efectos negativos para la salud. Algunas revisiones (EMCDDA, 2008; Hall y Degenhardt, 2009) han analizado los efectos adversos de su consumo, por ejemplo: los efectos agudos, como la ansiedad, reacciones de pánico o síntomas psicóticos; los efectos crónicos, como la dependencia o enfermedades respiratorias y los efectos en las funciones cognitivas.

Los consumidores de cannabis presentan importantes alteraciones funcionales en diversas áreas prefrontales, temporales y en el cerebelo, por lo que, en condiciones de activación, tienden a presentar patrones anormales de actividad en tareas cognitivas (Facundo, Martín-Santos, Abades, Farré y Verdejo-Garcia, 2008; Verdejo-Garcia, Pérez-Garcia, SánchezBarrera, Rodríguez-Fernández y Gómez-Río, 2007). En estos consumidores se aprecian alteraciones en la memoria (Jager, Kahn, Van Den Brink, Van Ree y Ramsey, 2006; Schwartz, Gruenewald, Klitzner y Fedio, 1986), atención (Chang, Yakupov, Cloak y Ernst, 2006), velocidad de procesamiento (Kelleher, Stough, Sergejew y Rolfe, 2004), coordinación motora (Pillay, Rogowska, Kanayama, Jon, Gruber, Simpson, Cherayil, Pope y Yurgelun-Todd, 2004), aprendizaje y control ejecutivo (inhibición, flexibilidad y toma de decisiones) (Fridberg et al., 2010; Grant, Chamberlain, Schreiber y Odlaug, 2011; Lundqvist, 2005; Verdejo, López-Torrecillas, Orozco y Pérez, 2002), lo que incide en una mala toma de decisiones (Fridberg et al., 2010).

Los efectos neuropsicológicos del cannabis han sido ampliamente analizados en las últimas décadas, sin embargo, existe un intenso debate en relación a la presencia de alteraciones temporales (limitados a las horas y días posteriores al consumo) (Fried, Watkinson y Gray, 2005; Pope, Gruber, Hudson, Huestis y Yurgelun-Todd, 2001) o deteriores estables (que permanecen durante períodos prolongados de abstinencia) (Bolla, Brown, Eldreth, Tate y Cadet, 2002; Schwartz, Gruenewald, Klitzner y Fedio, 1986), encontrándose resultados contradictorios. Para explicar la variabilidad de resultados, se han propuesto diversos factores que influyen en el rendimiento neuropsicológico, como puede ser la severidad del consumo (Bolla, Brown, Eldreth, Tate y Cadet, 2002; Bolla, Eldreth, Matochik y Cadet, 2005; Whitlow et al., 2004), o la edad de inicio del consumo (Slowij y Grenyer, 2002). Según varios estudios (Gruber, Rogowska y Yurgelun-Todd, 2009; Pope, Gruber, Hundon, Cohane, Huestis y YurgelunTodd, 2003; Wilson, Mathew, Turkington, Hawk, Coleman y Provenzale, 2000), el inicio del consumo en edades tempranas puede estar asociado a una mayor vulnerabilidad del sistema nervioso central a los efectos del cannabis, produciendo en estos individuos alteraciones más estables. También se han tenido en cuenta modelos matemáticos (Fridberg et al., 2010), comparando los resultados obtenidos por consumidores de cannabis con sujetos control en base a modelos probabilísticos de valencia, bien a través de expectativas de ganancia (EVL: Expectancy Valence Learnig), bien a través del rechazo a las pérdidas (PVL: Prospect Valence Learnig), siendo este segundo modelo un mejor predictor.

A pesar de que el uso crónico del cannabis se ha asociado con deterioros en diversos dominios cognitivos, varios estudios se centran en los efectos que tiene su uso en la toma de decisiones, ya que presenta un importante rol en la adicción y el abuso de las drogas (Whitlow et al., 2004). Rolls (2004), indica que la afectación de los procesos cognitivos involucrados en la toma de decisiones tiene una repercusión directa en la capacidad de la persona para desarrollar una vida independiente, autónoma y socialmente adaptada. La toma de decisiones es un proceso dinámico que favorece la elección, en situaciones de incertidumbre, de la alternativa más adecuada entre múltiples opciones de respuesta, valorando su influencia en futuras acciones (Clark, Cools y Robbins, 2004).

Una de las tareas más utilizadas para el estudio de la toma de decisiones en el contexto de la Hipótesis del Marcador Somático es la lowa Gambling Task (Bechara, Damasio, Damasio y Anderson, 1994), diseñada para estudiar los posibles déficit en los procesos de toma de decisiones de pacientes con daño neurológico en el área prefrontal ventromedial. Dicha Hipótesis fue propuesta por Damasio (1994/2004), en la que se describe la toma de decisiones como un proceso cognitivo en el que interviene la razón asistida por mecanismos de naturaleza emocional. Sugiere que la corteza prefrontal ventromedial actúa como zona de convergencia asociando una conducta con la emoción primaria provocada por su consecuencia. De tal forma, una situación concreta quedaría "marcada somáticamente" como agradable o desagradable en función de las consecuencias (positivas o negativas). En una nueva situación, similar a otra vivida anteriormente, la corteza prefrontal ventromedial activa una emoción secundaria (parecida a la experimentada en una situación análoga), provocando una emoción positiva o negativa en función de las experiencias previas. Este proceso de asociación se desarrolla de forma inconsciente, por lo que el sujeto experimenta una sensación acerca de la conducta en forma de intuición. Cuando este sistema falla, el sujeto no muestra ningún tipo de respuesta anticipatoria, y no es capaz de aprender de experiencias similares vividas 
con anterioridad, produciéndose una "miopía hacia el futuro." Esta incapacidad para generar marcadores somáticos se relaciona con la existencia de alteraciones neuropsicológicas en los procesos de toma de decisiones y la emisión de comportamientos socialmente inapropiados (Bechara, Damasio y Damasio, 2000).

La lowa Gambling Task (Bechara et al., 1994) simula situaciones de toma de decisiones de la vida diaria, en las cuales está la posibilidad de obtener recompensas 0 castigos en condiciones de incertidumbre y riesgo. En esta prueba, los sujetos eligen cartas de 4 mazos, y con cada elección se presentan ganancias monetarias junto con pérdidas ocasionales. Para su correcta ejecución, el sujeto debe seleccionar cartas de los mazos favorables (menores ganancias y menores pérdidas), y obviar aquellas de los mazos desfavorables, en los que las ganancias y penalizaciones son elevadas (Balodis, MacDonald y Olmstead, 2006; Dunn, Dalgleis y Lawrence, 2006; Fernie y Tunney, 2006; Lin, Chiu y Huang, 2009; Lin, Chiu, Lee y Hsieh, 2007). Numerosos estudios indican que en los pacientes con daño en la región ventromedial de la corteza prefrontal y en los sujetos drogodependientes (consumidores de alcohol, cocaina, heroína, metanfetaminas o cannabis), existe deterioro en la ejecución de dicha prueba. Tienden a elegir más cartas de los mazos desfavorables, sin embargo los sujetos sanos acaban seleccionando más cartas de los mazos favorables (Bechara, 2003; Bechara, Dolan, Denburg, Hindes, Anderson y Nathan, 2001; Bechara y Damasio, 2002; Grant, Contoreggi y London, 2000; Mazas, Finn y Steinmetz, 2000; Mogedas y Alameda, 2011; Shannon, Mathias, Dougherty y Liguori, 2010; Vélez, Borja y Ostrosky-Solís, 2010; Verdejo, Aguilar y Pérez-Garcia, 2004; Whitlow et al., 2004). No obstante los drogodependientes si pueden llegar a ser capaces de realizar la IGT de forma adecuada, ya que como muestra el trabajo de Verdejo-García, Benbrook, Funderbruk, David, Cadet y Bolla (2007) mejoran notablemente cuando la realizan por segunda vez, no tanto como los sujetos controles, y más que los consumidores de cocaína.

Bechara et al., (2001) propusieron que los sujetos drogodependientes manifestaban un patrón de conducta similar al de los pacientes con daño ventromedial: a menudo, niegan o no son conscientes de que tienen un problema, y se caracterizan por la tendencia a seleccionar recompensas inmediatas, ignorando las posibles consecuencias negativas del futuro (Bolla et al. 2005).

En los diversos estudios citados se describe que el abuso de cannabis está relacionado con déficit en la calidad de los procesos de toma de decisiones, por lo que este trabajo tiene como objetivo examinar el rendimiento de una muestra de consumidores de cannabis en una tarea sensible a la medición de los procesos de toma de decisiones (similar a la lowa Gambling Task), comparándolo con el rendimiento de una muestra control. Se administrarán dos versiones de dicha prueba (normal e inversa), y se estudiará si el rendimiento está influido por la edad, sexo, nivel de estudios, edad de inicio y cantidad del consumo (número de cigarros al día).

\section{Método}

\section{Participantes}

Participaron 82 personas (54 hombres y 28 mujeres), con edades comprendidas entre los 19 y los 53 años, divididos en dos grupos (consumidores de cannabis y controles) de 41 personas cada uno. Las características más relevantes de ambos grupos son las siguientes:

- Consumidores de cannabis: 27 (66\%) hombres y 14 (34\%) mujeres. Edad media de 24,66 años (SD $=5,637$, $M d n=24$ años) con un rango comprendido entre los 19 y los 50 años. En cuanto al nivel de estudios, 15 (36\%) cuentan con estudios primarios, 13 (32\%) con estudios secundarios y otros 13 (32\%) universitarios. La edad media en la que se iniciaron en el consumo fue de 17,39 años $(S D=5,454, M d n=16)$ con un rango entre los 13 y los 48 años. El consumo estaba comprendido entre 1 y 20 cigarrillos de cannabis al día con una media de 6 $(S D=3,556, M d n=6)$.

- Sujetos control: 27 (66\%) hombres y 14 (34\%) mujeres. Edad media de 25,17 años ( $S D=5,652, M d n=24$ años) con un rango de edad comprendido entre los 20 y los 53 años. En cuanto al nivel de estudios, 13 (32\%) participantes tienen estudios primarios, 13 (32\%) secundarios y 15 (36\%) universitarios. Ningún sujeto del grupo control había consumido algún tipo de drogas de forma regular.

Todos los participantes, tanto fumadores como controles fueron seleccionados mediante muestreo intencional y están vinculados a la Universidad de Huelva, como estudiantes de grado, licenciatura, másters y doctorado, personal de administración y servicios, o trabajadores de distintas contratas.

\section{Instrumento}

Se utilizó el programa "Cartas" (Palacios, Paíno y Alameda, 2010), una versión informatizada basada en la lowa Gambling Task (Bechara et al., 1994), para medir el proceso de toma de decisiones en situaciones de incertidumbre. El participante observa en la pantalla del ordenador cuatro mazos de cartas boca abajo y debe escoger libremente cartas con el ratón, previamente en las instrucciones se le indica que el juego consiste en intentar conseguir el máximo dinero posible. Cada mazo contiene 40 cartas, y la tarea consta de un total de 100 ensayos (esto es desconocido por el participante). Cada vez que se selecciona una carta, ésta se voltea y aparece un mensaje que indica la cantidad de dinero ganado y perdido y un signo que califica el resultado de la jugada, "+" si se incrementa el saldo, "-" si éste disminuye, e "=" si no varía, ajustándose la cantidad del saldo al resultado obtenido.

La peculiaridad de la tarea estriba en que hay dos mazos favorables ( $C$ y $D)$ y dos desfavorables $(A$ y $B)$ :

A y $B$ proporcionan altas recompensas económicas y altos castigos. El mazo A contiene premios de $100 €$ mientras que las pérdidas son de 100, 150, 200, 250, 300 y 350 euros, 
en cinco de cada 10 cartas. El mazo $B$ contiene premios de $100 €$ y una única pérdida de 1250 euros en cada ciclo de 10 elecciones. En cada ciclo de 10 jugadas se ganan $1000 €$ y se pierden $1250 €$.

C y D proporcionan ganancias más bajas, pero también castigos menores. El mazo $\mathrm{C}$ contiene premios de $50 €$ y castigos de 25, 50 y 75 euros en cinco de cada 10 cartas. El mazo $D$ contiene premios de $50 €$ y un único castigo de $250 €$ cada 10 cartas. Con estos mazos, en cada ciclo de 10 jugadas se ganan $500 €$ y se pierden $250 €$.

La puntuación de la tarea o índice gambling (IG) se obtiene al restar el número de elecciones de mazos desventajosos ( $A$ $+B)$, del número de elecciones de mazos ventajosos $(C+D)$, es decir $I G=(C+D)-(A+B)$. Este $I G$, se calcula para el total de la prueba, la parte inicial (50 primeros ensayos), la parte final (ensayos del 51 al 100) y por bloques de 20 ensayos para evaluar la evolución de la misma.

Los parámetros iniciales de la prueba son: Saldo Inicial: 2000€, No de jugadas: 100, Préstamo si saldo negativo: $500 €$, Retardo para ocultar la carta: 2 segundos, Colocación: ABCD y Gambling Parcial: 20. Todos los participantes realizan la prueba dos veces, una normal y otra invertida. En la tarea invertida se intercambia el programa de ganancias/ pérdidas de los distintos mazos, así, los mazos favorables (ganancias) ahora son $A$ y $B$, mientras que los desfavorables (pérdidas) son $C$ y $D$, en este caso los mazos favorables proporcionan mayores pérdidas a corto plazo pero con ganancias a largo plazo, frente a los otros dos mazos que ofrecen menores pérdidas a corto plazo y sin ganancias a largo plazo. Las puntuaciones IG en esta tarea se obtienen al restarle a las elecciones ventajosas las desventajosas, según la fórmula $I G i=(A+B)-(C+D)$. Valores positivos en el $I G$ indicarán que se realizó la tarea sin asumir riesgos y un valor negativo del IG que se han asumido muchos riesgos, o incluso que la ejecución fue deficiente.

\section{Procedimiento}

Los participantes fueron informados de los objetivos del estudio y participaron voluntariamente en una única sesión individual. Antes de la realización de la prueba se obtuvieron los datos relativos a edad, sexo, nivel de estudios e información sobre el consumo de drogas (edad de inicio y cantidad de consumo diaria). A continuación se administró la tarea normal e inversa, en orden aleatorizado.

Las evaluaciones se realizaron en una sala de la Universidad de Huelva, dotada de todo el instrumental necesario para el estudio, convenientemente iluminada y aislada acústicamente. La duración de la administración de la tarea fue de 15-20 minutos ininterrumpidos. Finalmente se proporcionó una explicación con los resultados obtenidos en la prueba a todos los participantes que lo solicitaron.

\section{Variables Utilizadas y Análisis Estadístico}

Los datos procedentes de la entrevista y de la tarea se trabajaron y analizaron con el SPSS.

Las variables dependientes utilizadas fueron:

- IG: las puntuaciones obtenidas por los participantes en los 100 ensayos de las tareas normal (IG) e inversa (IGi), los indices parciales asociados a las 50 primeras jugadas (normal: IGP e inversa: IGPi), y las 50 últimas jugadas (normal: IGF e inversa: IGFi), así como la puntuación obtenida en cada bloque de 20 ensayos, tanto en la tarea normal (IG20, IG40, IG60, IG80 e IG100) como en la inversa (IG20i, IG40i, IG60i, IG80i e IG100i)

- El número de cartas elegidas en cada mazo ( $A, B, C$ y $D)$, tanto en la tarea normal e inversa, como en el total y en los distintos parciales utilizados para la obtención del IG.

La variable independiente utilizada fue el consumo o no consumo de cannabis, utilizando como covariables la edad del participante, la edad de inicio al consumo y el número de cigarrillos de cannabis consumidos diariamente. El sexo y el nivel de estudios fueron considerados en los análisis como factores fijos. Se realizaron análisis estadísticos del total de la prueba y de los distintos parciales utilizados, tanto en la versión normal como inversa.

Para analizar la influencia del consumo/no consumo sobre el rendimiento de la tarea, se realizaron t de student; y para comparar la evolución a lo largo de los diferentes bloques, se utilizaron análisis de medidas repetidas.

\section{Resultados}

\section{Procedimiento Normal}

Diversos estudios utilizan un IG de 10 como indicador de la buena o mala ejecución en la tarea, precisamente porque ésta ha sido la puntuación máxima alcanzada por pacientes neurológicos con daño en el área ventromedial del córtex prefrontal (Bechara y Damasio, 2002; Bechara et al., 2001; Bechara et al., 2002), indicando que las puntuaciones inferiores a 10 corresponden a una mala ejecución, mientras que las puntuaciones superiores señalan una buena ejecución de la tarea. En este caso, dentro de los consumidores de cannabis, el 73\% de los participantes (30 de 41) presentan un IG inferior a 10, mientras que en los sujetos controles, estas puntuaciones inferiores a 10 se dan en 13 de 41 , es decir en el $31,7 \%$ de los participantes. Este dato ha de ser tomado como un mero indicador, ya que personas normales-sanas también obtienen puntuaciones inferiores a 10, sin que ello implique la existencia de problemas en la toma de decisiones por alteraciones en la corteza ventromedial.

En la Tabla 1 se representan los resultados obtenidos en las distintas variables, tanto de los consumidores de cannabis como de los sujetos controles. 
Tabla 1.- Estadísticos descriptivos de los distintos índices de la prueba.

\begin{tabular}{ccccc|cccc|cc} 
& \multicolumn{3}{c|}{ Fumadores de Cannabis } & \multicolumn{5}{c|}{ Controles } & \multicolumn{2}{c}{$\mathrm{t}(1-80)$} \\
\cline { 2 - 10 } & $\mathrm{M}$ & SD & Mdn & rango & $\mathrm{M}$ & SD & Mdn & rango & $\mathrm{t}$ & $\mathrm{p}$ \\
\hline IG & 5,71 & 18,047 & 2 & $-20 / 60$ & 19,12 & 16,799 & 18 & $-8 / 60$ & 3,48 &, 001 \\
IGP & 1,02 & 11,046 & 0 & $-18 / 32$ & 3,71 & 9,629 & 2 & $-16 / 26$ & 1,17 &, 245 \\
IGF & 4,68 & 11,529 & 2 & $-20 / 44$ & 15,41 & 14,340 & 12 & $-6 / 50$ & 3,73 &, 000 \\
IG20 &,- 976 & 5,7118 & 0 & $-12 / 16$ & $-2,146$ & 6,1382 & -2 & $-20 / 12$ & 0,89 &, 374 \\
IG40 & 1,80 & 5,582 & 2 & $-12 / 18$ & 3,02 & 7,773 & 4 & $-20 / 20$ & 0,81 &, 417 \\
IG60 &, 78 & 7,098 & -2 & $-10 / 20$ & 5,02 & 7,800 & 6 & $-18 / 20$ & 2,58 &, 012 \\
IG80 & 2,39 & 6,667 & 2 & $-12 / 20$ & 5,90 & 7,784 & 2 & $-6 / 20$ & 2,19 &, 031 \\
IG100 & 1,71 & 6,333 & 0 & $-10 / 16$ & 7,32 & 6,223 & 6 & $-4 / 20$ & 4,05 &, 000 \\
\hline
\end{tabular}

Como se puede apreciar en todos los indicadores (salvo en el IG20) los resultados obtenidos por los sujetos controles son mayores. Las diferencias entre los IG de ambos grupos se ciñen a la parte final, ya que en las primeras 50 jugadas las diferencias no son estadísticamente significativas, mientras si lo son en las 50 jugadas finales, además, en los fumadores de cannabis no hay diferencias entre la fase inicial de la prueba y la fase final $\left(t_{(1-40)}=1,726, p=, 092\right)$, diferencias que si son significativas en el caso de los sujetos controles $\left(t_{(1-40)}=4,227, p=, 000\right)$. En cuanto al análisis por bloques hay diferencias estadisticamente significativas en los tres últimos bloques, es decir hasta la jugada 40 la ejecución en ambos grupos es similar, pero a partir de ésta la ejecución de los controles es mejor (Figura 1).

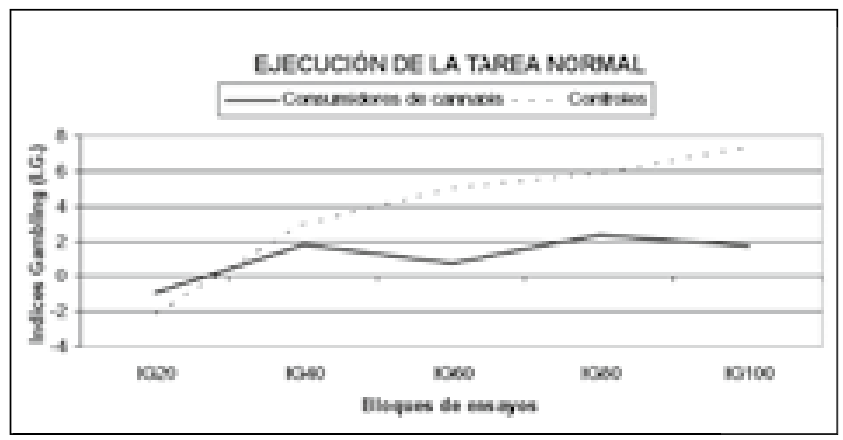

Figura 1.- Índice Gambling por bloques para los consumidores de cannabis y el grupo control.

Como muestra la Figura 1, la ejecución de la tarea en los sujetos consumidores es prácticamente plana en los cinco bloques, sin embargo, los sujetos controles muestran una línea ascendente. Los análisis de medidas repetidas nos muestran diferencias estadísticamente significativas $\left(F_{(4-77)}=\right.$ $8,951, p=, 000)$, siendo también significativas las diferencias encontradas entre los fumadores de cannabis y los controles como factor intersujetos $\left(F_{(4-77)}=3,022, p=, 023\right)$. Si analizamos el desarrollo de la prueba de forma separada para ambos grupos, podemos observar que no hay diferencias en la evolución de la tarea a lo largo de los cinco bloques en los consumidores de cannabis $\left(F_{(4-37)}=2,330, p=, 074\right)$, no obstante si se obtienen diferencias estadísticamente signi- ficativas entre los dos primeros bloques, IG20-IG40 $\left(t_{(1-40)}\right.$ $=2,350, p=, 024)$ y entre el primero y el cuarto, IG20-IG80 $\left(t_{(1-40)}=2,350, p=, 006\right)$. La tendencia ascendente observada en los sujetos control si se ve confirmada $\left(F_{(4-37)}=9,086\right.$, $p=, 000)$, si bien las diferencias significativas entre bloques se centran en la parte inicial de la prueba, entre el primer bloque y el resto (IG20-IG40: $t_{(1-40)}=3,109, p=, 003 ;$ IG20-IG60: $t_{(1-40)}=4,068, p=, 000 ;$ IG20-IG80: $t_{(1-40)}=4,719, p=, 000$; IG20-IG100: $t_{(1-40)}=6,078, p=, 000$;) y entre el IG40-IG100 $\left(t_{(1-40)}=3,130, p=, 003\right)$. A partir del IG60, a pesar de mantener una línea ascendente no hay diferencias significativas con respecto a los bloques finales (IG80 e IG100).

En cuanto al número de elecciones de cada mazo, presentamos los resultados en la Tabla 2. Ambos grupos muestran una preferencia por el mazo D (favorable), siendo el que recibe el mayor número de elecciones en el cómputo global de la prueba y en la parte final; del mismo modo, ambos grupos seleccionan en menor medida el mazo A (desfavorable), en el cómputo total de la prueba y en la parte final. En la fase inicial, ambos grupos coinciden en su rechazo al mazo $A$, mientras que la preferencia de los consumidores de cannabis es el mazo B y la de los controles el C. Si analizamos las diferencias de medias entre ambos grupos en el número de elecciones de cada mazo, vemos que hay coincidencia en las elecciones del mazo $D$, ya que tanto en el cómputo global de la prueba como en las partes inicial y final, las diferencias no son significativas. En cuanto a las elecciones del mazo $A$, las diferencias observadas sólo son estadisticamente significativas en el cómputo global de la prueba. Las diferencias observadas en el mazo $B$, son estadísticamente significativas en el cómputo global de la prueba y en la parte final, y por último en el número de elecciones del mazo $\mathrm{C}$ las diferencias son estadísticamente significativas, tanto en el cómputo global de la prueba como en las partes inicial y final.

Tabla 2.- Número de elecciones de cada mazo, en la prueba total y en la parte inicial y final de la misma.

\begin{tabular}{lccc|ccc|cc} 
& \multicolumn{2}{c}{ Fumadores Cannabis } & \multicolumn{3}{c|}{ Controles } & \multicolumn{2}{c}{$t(1-80)$} \\
\cline { 2 - 8 } & $\mathrm{M}$ & $\mathrm{SD}$ & rango & $\mathrm{M}$ & $\mathrm{SD}$ & rango & $\mathrm{t}$ & $\mathrm{p}$ \\
\hline $\mathrm{nA}^{1}$ & 18,78 & 6,126 & $10-33$ & 15,95 & 5,731 & $7-32$ & 2,160 &, 034 \\
$\mathrm{nB}^{1}$ & 28,37 & 7,562 & $7-40$ & 24,49 & 6,874 & $11-40$ & 2,430 &, 017 \\
$\mathrm{nC}^{1}$ & 24,37 & 6,726 & $12-40$ & 29,61 & 7,334 & $16-40$ & $-3,374$ &, 001 \\
$\mathrm{nD}^{1}$ & 28,49 & 7,008 & $14-40$ & 29,95 & 6,618 & $16-40$ & $-0,971$ &, 334 \\
$\mathrm{nA}^{\mathrm{P}}$ & 10,22 & 3,482 & $4-20$ & 9,29 & 2,918 & $0-15$ & 1,306 &, 195 \\
$\mathrm{nBP}^{\mathrm{n}}$ & 14,27 & 3,975 & $4-21$ & 13,85 & 4,234 & $5-23$ & 0,457 &, 649 \\
$\mathrm{nC}^{\mathrm{P}}$ & 12,15 & 3,870 & $4-25$ & 14,12 & 4,354 & $7-25$ & $-2,171$ &, 033 \\
$\mathrm{nD}^{\mathrm{P}}$ & 13,37 & 4,420 & $7-27$ & 12,73 & 4,914 & $0-24$ & 0,614 &, 541 \\
$\mathrm{nA}^{\mathrm{F}}$ & 8,56 & 4,178 & $1-16$ & 6,66 & 4,710 & $0-21$ & 1,935 &, 057 \\
$\mathrm{nB}^{\mathrm{F}}$ & 14,10 & 5,389 & $0-26$ & 10,63 & 5,412 & $0-19$ & 2,904 &, 005 \\
$\mathrm{nC}^{\mathrm{F}}$ & 12,22 & 4,269 & $3-23$ & 15,49 & 5,320 & $6-29$ & 3,068 &, 003 \\
$\mathrm{nD}^{\mathrm{F}}$ & 15,12 & 5,391 & $6-32$ & 17,22 & 5,769 & $5-35$ & 1,701 &, 093 \\
\hline
\end{tabular}

NOTA: ${ }^{1}$ hace referencia al número de elecciones de cada mazo en toda la prueba: ${ }^{\mathrm{P}}$, hace referencia a las 50 primeras jugadas (parte inicial); $y^{*}$, hace referencia a la parte final de la prueba, es decir, las últimas 50 jugadas. 
Analizando las estrategias de elección de mazos en ambos grupos obtenemos los resultados que se muestran en la Tabla 3. El dato a destacar es que no debería haber diferencias significativas entre las elecciones de los mazos favorables ( $C$ y D), lo cual se cumple en los controles y no en los consumidores de cannabis (salvo en la fase inicial del juego), ni tampoco entre los mazos desfavorables ( $A$ y $B$ ) aspecto que no cumplen ninguno de los dos grupos. Este dato se complementa con el hecho de que en los consumidores de cannabis no hay diferencias en las elecciones del mazo $B$ con respecto a las elecciones de los mazos $C$ y $D$, circunstancia que no se da en la fase final y global en los controles.

Tabla 3.- Diferencias de medias en las estrategias de elección de mazos en ambos grupos.

\begin{tabular}{|c|c|c|c|c|c|c|c|c|c|c|c|c|}
\hline & \multicolumn{6}{|c|}{ Consumidores de Cannabis } & \multicolumn{6}{|c|}{ Controles } \\
\hline & \multicolumn{2}{|c|}{ Global } & \multicolumn{2}{|c|}{ Principio } & \multicolumn{2}{|c|}{ Final } & \multicolumn{2}{|c|}{ Global } & \multicolumn{2}{|c|}{ Principio } & \multicolumn{2}{|c|}{ Final } \\
\hline & $\mathrm{t} 1-40$ & $p$ & t1-40 & $p$ & t1-40 & $p$ & $\mathrm{t} 1-40$ & $p$ & t1-40 & $p$ & $\mathrm{t} 1-40$ & $p$ \\
\hline$n A-n B$ & $-5,906$ &, 000 & $-5,149$ &, 000 & $-4,586$ &, 000 & $-5,773$ &, 000 & $-5,359$ &, 000 & $-3,546$ &, 001 \\
\hline$n A-n C$ & $-3,555$ &, 001 & $-1,994$ &, 053 & $-4,064$ &, 000 & $-8,355$ &, 000 & $-5,783$ & ,000 & $-6,762$ &, 000 \\
\hline$n A-n D$ & $-5,452$ &, 000 & $-2,928$ &, 006 & $-5,000$ &, 000 & $-8,553$ &, 000 & $-3,272$ & ,002 & $-7,544$ &, 000 \\
\hline$n B-n C$ & 2,015 &, 051 & 2,013 &, 051 & 1,416 & 164 & $-2,604$ &, 013 &,- 234 &, 816 & $-3,378$ &, 002 \\
\hline$n B-n D$ &,- 064 &, 949 & ,781 & ,439 &,- 742 &, 462 & $-3,215$ & ,003 & ,945 & 350 & $-4,348$ & , 000 \\
\hline$n C-n D$ & $-2,548$ &, 015 & $-1,258$ & 216 & $-2,373$ &, 023 & -196 & ,846 & 1,121 & 269 & $-1,309$ & 198 \\
\hline
\end{tabular}

NOTA: $n=$ número de elecciones de cada mazo en la tarea normal

Si tenemos en cuenta la evolución de las elecciones de mazos a lo largo de los cinco bloques obtenemos unos resultados similares. En la Figura 2 se puede apreciar cómo en las distintas fases de la prueba las elecciones del mazo $B$ de los consumidores de cannabis siempre están por encima de las elecciones del mazo $C$, en cambio en los sujetos controles, aunque las elecciones de $B$ son más elevadas de lo que cabría esperar, éstas están por debajo de las elecciones de $C$, en todos los bloques salvo en el primero.

Para facilitar la comprensión de las elecciones, podemos agrupar los mazos en dos grupos y diferenciar entre elecciones ventajosas $(C+D)$ y desventajosas $(A+B)$. En este caso se puede apreciar que existen diferencias estadísticamente significativas entre las elecciones de ambos grupos, si bien, en el primer bloque de la prueba son más las elecciones desventajosas que las ventajosas (11 frente a $9, t_{(1-81)}=$ $2,387 ; p=, 019)$, en el resto de bloques hay predilección por las ventajosas. Sin embargo los resultados en los fumadores de cannabis y controles son diferentes, en los primeros sólo son significativas las diferencias del segundo bloque -IG40(11 favorables frente a 9 desfavorables: $t_{(1-40)}=2,070$; $p=, 045)$ y el cuarto bloque -IG80- (12 favorables frente a 8 desfavorables: $\left.t_{(1-40)}=2,296 ; p=, 027\right)$, mientras que en los controles la predilección por las ventajosas es mucho más clara y progresiva, así, en el segundo bloque seleccionan 12 favorables frente a 8 desfavorables $\left(t_{(1-40)}=2,491 ; p=, 017\right)$, en el tercer y cuarto bloque seleccionan 13 favorables frente a 7 desfavorables $\left(t_{(1-40)}=4,139 ; p=, 000\right.$ y $t_{(1-40)}=4,855$; $p=, 000$; respectivamente), y en el último bloque 14 favorables y 6 desfavorables $\left(t_{(1-40)}=7,529 ; p=, 000\right)$.
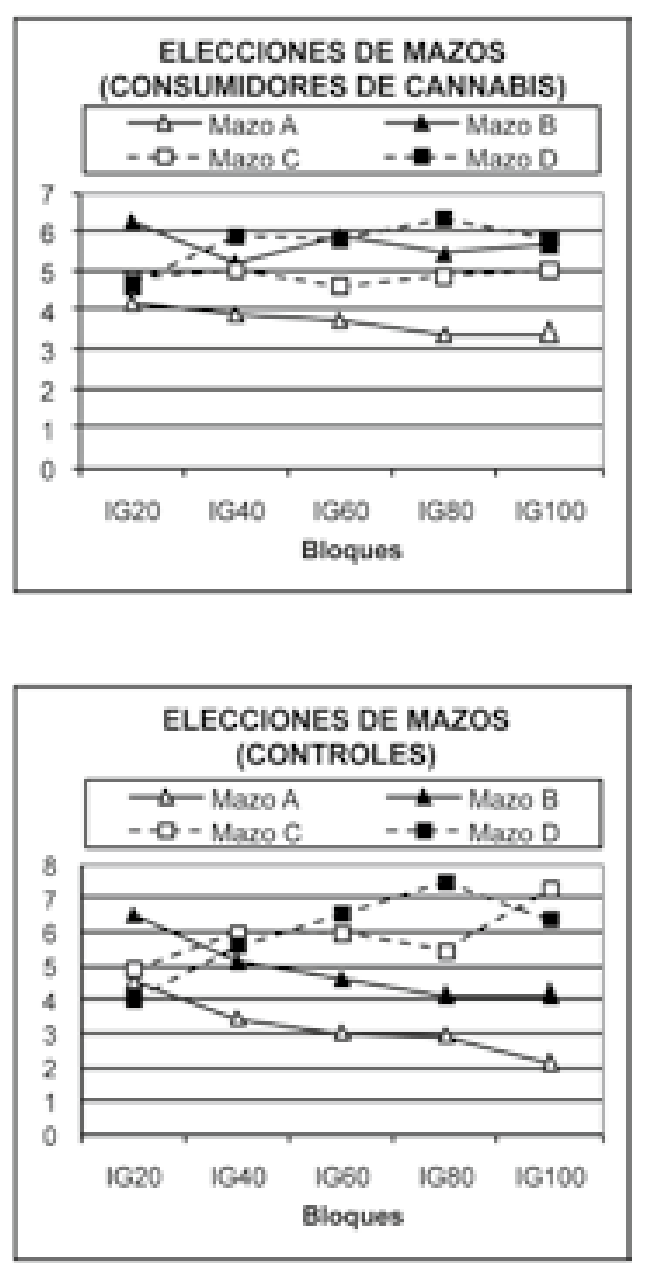

Figura 2.- Elecciones de mazos a lo largo de los 5 bloques en los consumidores de cannabis (arriba) y sujetos controles (abajo).

En el análisis por bloques de la incidencia de la edad, el sexo y el nivel de estudios hemos obtenido los siguientes resultados:

- Edad: no incide en los resultados obtenidos con todos los participantes $\left(F_{(4-76)}=0,318, p=, 865\right)$, ni de forma separada en los fumadores de cannabis $\left(F_{(4-36)}=0,299\right.$, $p=, 877)$, y en los sujetos control $\left(F_{(4-36)}=0,339, p=, 850\right)$.

- Sexo: no influye $\left(F_{(4-75)}=0,834, p=, 508\right)$, ni en los controles $\left(F_{(4-36)}=1,295, p=, 260\right)$, ni en los fumadores de cannabis $\left(F_{(4-36)}=0,318, p=, 865\right)$ aunque en éstos, si hay una interacción entre el sexo y el nivel de estudios $\left(F_{(4-30)}\right.$ $=3,556, p=, 002$ ), ya que los hombres sin estudios tienden a tener mejores puntuaciones que el resto, mientras que en las mujeres son la universitarias las que tienden a presentar mejores puntuaciones.

- Nivel de estudios: no hay influencia con todos los sujetos $\left(F_{(8-73)}=0,677, p=, 707\right)$, ni en el grupo de fumadores $\left(F_{(4-36)}=1,022, p=, 428\right)$, pero si en los controles $\left(F_{(4-36)}=\right.$ $2,289, p=, 031)$, ya que los universitarios tienden a presentar mejores puntuaciones, dato que en los fumadores de cannabis se da sólo en las mujeres. 
La influencia de la edad, el sexo y el nivel de estudios no resulta significativa $(p>, 05)$ ni en el IG total, ni en el IGP (50 primeras jugadas), ni en el IGF (últimas 50 jugadas).

Por último, en el caso de los fumadores de cannabis, hemos podido observar que ni la edad de inicio al consumo, ni la cantidad consumida a diario son factores significativos en ninguno de los índices analizados.

\section{Procedimiento Inverso}

Consiste en intercambiar el programa de pérdidas y ganancias, es decir pasar las pérdidas a ganancias y viceversa. El objetivo es comprobar si los resultados que se obtienen en el primer experimento se deben a la insensibilización a las pérdidas, si bien, un dato previo a tener en cuenta es que tras una pérdida de $1250 €$ los controles tardan una media de 6,2 jugadas en volver a seleccionar $B$, mientras que los fumadores de cannabis tardan una media de 5 jugadas, lo que ya nos indica que hay sensibilidad a las pérdidas.

En Tabla 4 se representan los resultados obtenidos en las distintas variables, tanto de los consumidores de cannabis como de los sujetos controles.

Tabla 4.- Estadísticos descriptivos de los distintos índices de la prueba en la modalidad inversa.

\begin{tabular}{lcccc|cccc|cc} 
& \multicolumn{3}{c|}{ Fumadores de Cannabis } & \multicolumn{5}{c|}{ Controles } & \multicolumn{2}{c}{$\mathrm{t}(1-80)$} \\
\cline { 2 - 11 } & $\mathrm{M}$ & SD & Mdn & rango & $\mathrm{M}$ & SD & Mdn & rango & $\mathrm{t}$ & $\mathrm{p}$ \\
\hline IGi & 16,63 & 20,501 & 8 & $-26 / 60$ & 0,98 & 25,745 & 4 & $-60 / 48$ & 3,047 &, 003 \\
IGPi & 9,90 & 13,813 & 6 & $-24 / 38$ & $-2,29$ & 17,712 & 2 & $-46 / 30$ & 3,477 &, 001 \\
IGFi & 6,73 & 12,812 & 6 & $-18 / 46$ & 3,27 & 17,960 & 2 & $-40 / 38$ & 1,005 &, 318 \\
IG20i & 1,56 & 6,173 & 2 & $-10 / 20$ & $-1,56$ & 8,432 & -2 & $-20 / 20$ & 1,913 &, 059 \\
IG40i & 5,56 & 7,570 & 4 & $-8 / 20$ & $-1,51$ & 10,524 & -2 & $-20 / 20$ & 3,494 &, 001 \\
IG60i & 4,83 & 7,416 & 4 & $-8 / 20$ & 0,98 & 11,884 & 2 & $-20 / 20$ & 1,762 &, 082 \\
IG80i & 4,54 & 7,086 & 4 & $-8 / 20$ & 1,61 & 9,646 & 0 & $-20 / 20$ & 1,566 &, 121 \\
IG100i & 0,15 & 7,421 & 0 & $-14 / 20$ & 1,46 & 10,286 & 0 & $-20 / 20$ & 0,665 &, 508 \\
\hline
\end{tabular}

Como se puede apreciar en la Tabla 4, el IGi de los consumidores de cannabis es muy superior al de los controles, diferencia que es además estadisticamente significativa. Sin embargo, si analizamos las jugadas iniciales (IGiP) y las finales (IGiF), vemos que las diferencias se ciñen a la parte inicial de la prueba, ya que en las últimas 50 jugadas no hay diferencias significativas entre consumidores de cannabis y sujetos controles. Además, no hay diferencias entre la fase inicial y final de la prueba, ni en los fumadores de cannabis $\left(t_{(1-40)}=1,193, p=, 240\right)$, ni en los sujetos controles $\left(t_{(1-40)}=\right.$ $1,442, p=, 157)$. Esta tendencia se da también en el análisis por bloques ya que salvo en el segundo bloque no hay diferencias significativas entre ambos grupos, si bien la diferencia que se produce entre los grupos en el primer bloque está cerca de ser significativa.

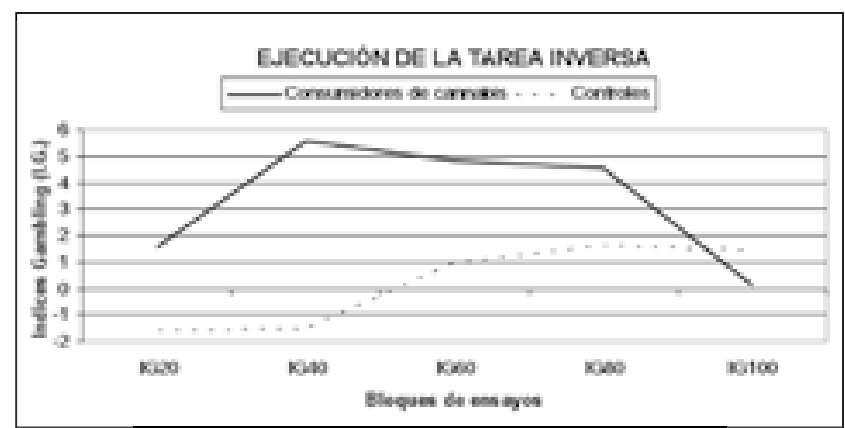

Figura 3.- Índice Gambling por bloques para los consumidores de cannabis y el grupo control en la prueba inversa.

Como se puede apreciar en la Figura 3, la ejecución de la tarea en los sujetos consumidores es inicialmente mejor que la de los sujetos controles a lo largo de los 4 bloques iniciales, para igualarse en el último, sin embargo, tal como se muestra en la tabla 4, las diferencias entre ambos grupos se ciñen sólo al segundo bloque. Los análisis de medidas repetidas no muestran diferencias significativas $\left(F_{(4-77)}=\right.$ $2,094, p=, 090)$, tampoco resultan significativas las diferencias entre los fumadores de cannabis y los sujetos control como factor intersujetos $\left(F_{(4-77)}=1,864, p=, 125\right)$. Si analizamos el desarrollo de la prueba de forma separada para ambos grupos, podemos observar que hay diferencias en la evolución de la ejecución de la tarea a lo largo de los cinco bloques en los consumidores de cannabis $\left(F_{(4-37)}=4,728, p=\right.$ $, 003)$, se obtienen diferencias estadisticamente significativas entre el primer bloque -IGi20-y el $2^{\circ}-I G i 40-\left(t_{(1-40)}=3,066\right.$, $p=, 004), 3^{\circ}-$ IGi60- $\left(t_{(1-40)}=2,260, p=, 029\right)$ y $4^{\circ}-$ IGi80$\left(t_{(1-40)}=2,206, p=, 033\right)$ y entre el bloque $2^{\circ}-I G i 40-y$ el $5^{\circ}-$ IGi100- $\left(t_{(1-40)}=3,258, p=, 002\right)$, lo que confirma la tendencia descendente de la fase final de la prueba en los consumidores de cannabis.

En cuanto a la evolución de la tarea en los sujetos controles, ésta no muestra diferencias significativas $\left(F_{(4-37)}=\right.$ $0,853, p=, 501)$, no habiendo tampoco diferencias significativas entre ninguno de sus bloques $(p>, 05)$, lo que denota una ejecución plana, a pesar de la tendencia ascendente que refleja la Figura 3.

En cuanto al número de elecciones de cada mazo (Tabla 5), ambos grupos muestran un mayor número de elecciones del mazo A (favorable) en el computo global de la prueba, mientras que los mazos menos elegidos son $D$ (desfavorable) en los fumadores de cannabis y B (favorable) en los sujetos controles; destacar que en ambos grupos el mazo C (desfavorable) es el segundo en orden de preferencia. Si analizamos las diferencias de medias entre las elecciones de mazos de ambos grupos, sólo es significativa las elecciones de D.

Si analizamos el patrón de elecciones de las 50 primeras jugadas podemos observar que en los fumadores de cannabis hay preferencia por el mazo A y el C, mientras que los controles prefieren $C$ y $A$. Los fumadores de cannabis presentan menor número de elecciones en $D$ y $B$, mientras que los controles lo hacen en $B$ y D. Las diferencias entre las elecciones de ambos grupos en esta fase son estadisticamente significativas, salvo las elecciones del mazo B. 
Tabla 5.- Número de elecciones de cada mazo en la prueba total, parte inicial y final de la versión inversa.

\begin{tabular}{cccc|ccc|cc} 
& \multicolumn{2}{c}{ Fumadores Cannabis } & \multicolumn{3}{c|}{ Controles } & \multicolumn{2}{c}{$\mathrm{t}(1-80)$} \\
\cline { 2 - 8 } & $\mathrm{M}$ & $\mathrm{SD}$ & $\mathrm{Mdn}$ & $\mathrm{M}$ & $\mathrm{SD}$ & Rango & $\mathrm{t}$ & $\mathrm{p}$ \\
\hline $\mathrm{nAi}^{1}$ & 33,56 & 8,322 & $11-40$ & 29,88 & 9,543 & $8-40$ & 1,863 &, 066 \\
$\mathrm{nBi}^{1}$ & 24,76 & 7,806 & $6-40$ & 20,61 & 10,954 & $2-40$ & 1,974 &, 052 \\
$\mathrm{nCi}^{1}$ & 25,49 & 6,867 & $11-40$ & 28,80 & 10,095 & $7-40$ & $-1,740$ &, 086 \\
$\mathrm{nDi}^{1}$ & 16,20 & 6,672 & $3-28$ & 20,71 & 8,180 & $3-40$ & $-2,737$ &, 008 \\
$\mathrm{nAi}^{\mathrm{P}}$ & 18,29 & 7,342 & $7-36$ & 13,98 & 7,866 & $1-28$ & 2,569 &, 012 \\
$\mathrm{nBi}^{\mathrm{P}}$ & 11,66 & 4,139 & $3-25$ & 9,88 & 5,492 & $1-21$ & 1,658 &, 101 \\
$\mathrm{nCi}^{\mathrm{P}}$ & 11,73 & 4,528 & $4-29$ & 14,98 & 7,350 & $1-38$ & $-2,406$ &, 018 \\
$\mathrm{nDi}^{\mathrm{P}}$ & 8,32 & 3,704 & $1-15$ & 11,17 & 4,934 & $1-25$ & $-2,962$ &, 004 \\
$\mathrm{nAi}^{\mathrm{F}}$ & 15,27 & 5,445 & $4-31$ & 15,90 & 7,870 & $3-37$ &,- 424 &, 672 \\
$\mathrm{nBi}^{\mathrm{F}}$ & 13,10 & 6,160 & $1-30$ & 10,73 & 8,056 & $0-31$ & 1,494 &, 139 \\
$\mathrm{nCi}^{\mathrm{F}}$ & 13,76 & 5,049 & $2-29$ & 13,83 & 6,285 & $1-27$ &,- 058 &, 954 \\
$\mathrm{nDi}^{\mathrm{F}}$ & 7,88 & 3,770 & $0-15$ & 17,22 & 5,622 & $0-21$ & $-1,569$ &, 121 \\
\hline
\end{tabular}

NOTA: ${ }^{1}$ hace referencia al número de elecciones de cada mazo en toda la prueba; ${ }^{\mathrm{P}}$, hace referencia a las 50 primeras jugadas (parte inicial); $\mathrm{Y}^{\mathrm{F}}$, hace referencia a la parte final de la prueba, es decir, las últimas 50 jugadas.

En la fase final, no hay diferencias significativas en las estrategias de elección de los mazos entre ambos grupos, se mantiene la misma estrategia en los fumadores de cannabis, en cambio, los sujetos controles, en esta parte final, muestran preferencia por $\mathrm{D}$ y $\mathrm{A}$ y rechazo por $\mathrm{B}$ y $\mathrm{C}$.

Analizando las estrategias de elección de mazos de ambos grupos (Tabla 6), destacar que no debería haber diferencias significativas entre las elecciones de los mazos favorables, (A y B), ni tampoco entre los mazos desfavorables (C y D). Otro aspecto a destacar son las diferencias que sí deberian obtenerse y que sin embargo no se dan, como es el caso de $B$ y $C$ en los fumadores de cannabis, o el de $A$ y $C$ en los sujetos controles.

Tabla 6. Diferencias de medias en las estrategias de elección de mazos en ambos grupos (tarea inversa).

\begin{tabular}{|c|c|c|c|c|c|c|c|c|c|c|c|c|}
\hline & \multicolumn{6}{|c|}{ Consumidores de Cannabis } & \multicolumn{6}{|c|}{ Controles } \\
\hline & \multicolumn{2}{|c|}{ Global } & \multicolumn{2}{|c|}{ Principio } & \multicolumn{2}{|c|}{ Final } & \multicolumn{2}{|c|}{ Global } & \multicolumn{2}{|c|}{ Principio } & \multicolumn{2}{|c|}{ Final } \\
\hline & $\mathrm{t} 1-40$ & $p$ & $\mathrm{t} 1-40$ & $p$ & t1-40 & $p$ & t1-40 & p & t1-40 & p & t1-40 & $p$ \\
\hline$n A i-n B i$ & 4,524 &, 000 & 4,372 &, 000 & 1,433 & 160 & 3,706 &, 001 & 2,553 & ,015 & 2,517 &, 016 \\
\hline$n A i-n C i$ & 4,209 &, 000 & 3,850 &, 000 & 1,111 & 273 & .426 &, 672 &,- 457 &, 650 & 1,072 &, 290 \\
\hline$n A i-n D i$ & 8,011 & ,000 & 6,161 & ,000 & 6,332 & ,000 & 3,894 & ,000 & 1,671 & , 103 & 3,731 & ,001 \\
\hline$n B i-n C i$ &,- 348 &, 730 &,- 068 & ,946 &,- 433 & ,668 & $-2,784$ &, 008 & $-3,091$ &, 004 & $-1,670$ & 103 \\
\hline$n B i-n D i$ & 4,827 &, 000 & 3,786 & ,001 & 3,997 &, 000 &,- 040 & 969 &,- 963 &, 341 &, 639 &, 526 \\
\hline$n C i-n D i$ & 6,726 &, 000 & 4,800 & ,000 & 6,077 & ,000 & 3,954 & ,000 & 2,753 &, 009 & 3,503 & ,001 \\
\hline
\end{tabular}

NOTA: $n=$ número de elecciones de cada mazo en la tarea inversa.

Si tenemos en cuenta la evolución de las elecciones de mazos a lo largo de los cinco bloques obtenemos unos resultados similares. En la Figura 4 se puede apreciar cómo en las distintas fases de la prueba los fumadores de cannabis selec- cionan mayoritariamente el mazo A mientras que en los sujetos controles esta preferencia se distribuye entre los mazos $A$ (favorable) y $\mathrm{C}$ (desfavorable).

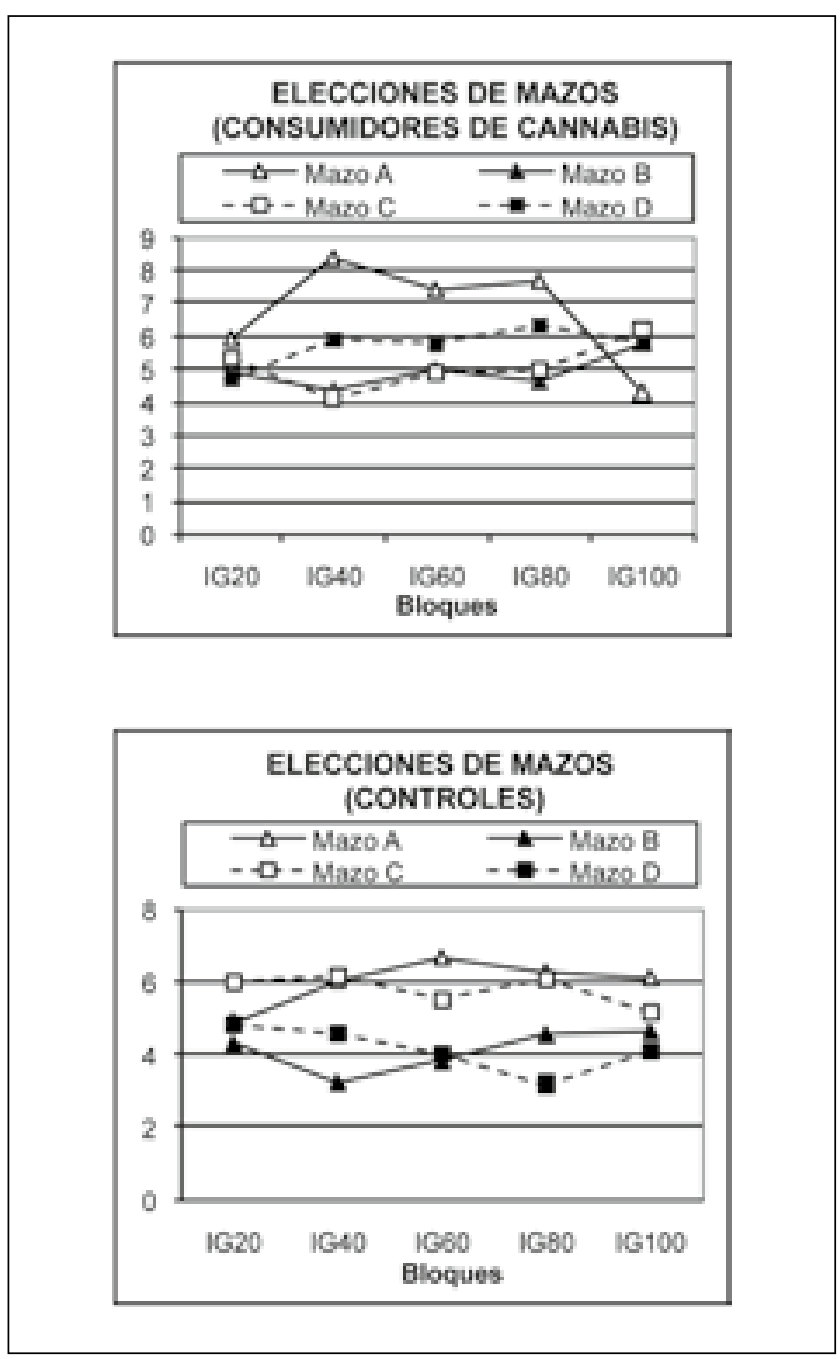

Figura 4.- Elecciones de mazos a lo largo de los 5 bloques en los consumidores de cannabis (arriba) y sujetos controles (abajo) en la versión inversa.

Agrupando los cuatro mazos en dos y diferenciando entre elecciones ventajosas $(A+B)$ y desventajosas $(C+D)$, podemos apreciar que existen diferencias estadísticamente significativas entre las elecciones de ambos tipos de mazos sólo en los bloques tercero -IG60- (11 favorables frente a 9 desfavorables: $\left.t_{(1-40)}=2,620 ; p=, 011\right)$ y cuarto - IG80(12 favorables frente a 8 desfavorables: $\mathrm{t}_{(1-40)}=3,259 ; p=$ ,002). Sin embargo los resultados en los fumadores de cannabis y controles son diferentes, ya que en los primeros son significativas las diferencias del segundo bloque -IG40- (13 favorables frente a 7 desfavorables: $\left.t_{(1-40)}=4,704 ; p=, 000\right)$, el tercer bloque -IG60- (12 favorables frente a 8 desfavorables: $\left.t_{(1-40)}=4,170 ; p=, 000\right)$ y el cuarto bloque -IG80- (12 favorables frente a 8 desfavorables: $\left.t_{(1-40)}=4,100 ; p=, 000\right)$, mientras que en los controles no hay ninguna predilección a lo largo de los distintos bloques. 
La incidencia de edad y el sexo no resulta significativa en el desarrollo de la tarea en los distintos IG $(p>, 05)$. En cambio, el nivel de estudios si resulta significativo en el IGi total en los fumadores de cannabis $\left(F_{(2-34)}=5,314, p=, 010\right)$, donde los sujetos con estudios primarios presentan los IGi más bajos. Se presenta también una interacción entre nivel de estudios y sexo, tanto en el IGi $\left(F_{(2-34)}=4,376, p=, 020\right)$ como en el IGiF $\left(F_{(2-34)}=4,707, p=, 016\right)$ en fumadores de cannabis y en el IGiP $\left(F_{(2-34)}=5,129, p=, 011\right)$ de los sujetos controles, ya que las mujeres presentar mejores puntuaciones que los hombres y, además, en las mujeres las mejores puntuaciones se corresponden con las universitarias y en los hombres, con los que tienen estudios secundarios. No obstante estos datos deben ser tomados con prudencia ya que el $66 \%$ de los participantes son hombres y el $34 \%$ mujeres.

Por último, en el caso de los fumadores de cannabis, hemos podido observar que ni la edad de inicio al consumo, ni la cantidad consumida a diario son factores significativos en ninguno de los índices analizados $(p>, 05)$.

\section{Discusión}

El objetivo del presente estudio era evaluar el proceso de toma de decisiones en consumidores de cannabis, en el contexto de la Hipótesis del Marcador Somático, a través del programa "Cartas" (similar a la lowa Gambling Task). Para ello, se comparó el rendimiento de los consumidores de cannabis con un grupo de no consumidores, en la versión normal e inversa de la tarea, y se analizó la influencia de la edad, sexo, nivel de estudios, edad de inicio y cantidad del consumo (número de cigarros al día).

En relación a la ejecución de la tarea normal, los resultados muestran que existen diferencias estadisticamente significativas entre el grupo control y el experimental en el IG total, obteniendo puntuaciones inferiores los consumidores de cannabis, lo que puede indicar la presencia de déficit o deterioro en el proceso de toma de decisiones, datos que están en la línea de otras investigaciones (Bolla et al., 2005; Facundo et al., 2008; Fridberg et al., 2011; Verdejo et al., 2004; Whitlow et al., 2004).

En el análisis por bloques, para analizar la evolución del aprendizaje de la tarea, existen diferencias estadísticamente significativas entre ambos grupos en los tres últimos bloques (IG60, IG80 e IG100), así como en el IG-Final, pero no en los dos primeros bloques (IG20 e IG40) ni en el IG-Inicial (50 primeros ensayos). Esto puede ser debido a que en el inicio de la prueba, ambos grupos están sondeando las características de los cuatro mazos. Tal y como indican Dunn, Dalgleish y Lawrence (2006), la elección al inicio de la prueba de cartas desfavorables no puede interpretarse como un déficit en la toma de decisiones, sino como el resultado lógico del proceso de exploración que se realiza al principio de la misma. En los controles se observa una tendencia claramente ascendente, produciéndose un incremento de las puntuaciones a lo largo de la tarea, lo que indica un patrón de toma de decisio- nes favorables. Esta tendencia no se aprecia en los consumidores de cannabis, en el inicio de la prueba (IG20) obtienen puntuaciones ligeramente superiores a las del grupo control, pero no se produce un incremento de las mismas a lo largo de su ejecución, lo que indica un patrón de toma de decisiones desfavorables. Estos resultados son consistentes con investigaciones previas que demuestran una relación entre el abuso de sustancias y alteraciones en el proceso de toma de decisiones, reforzando así la aplicación de la hipótesis del marcador somático al estudio de las adicciones (v.g. Bechara et al., 2002; Bechara et al., 2001; Bechara y Damasio, 2002; Bolla et al., 2005; Grant et al., 2000; Mogedas y Alameda, 2011; Vélez et al., 2010; Verdejo-García et al., 2007; Verdejo et al., 2002; Whitlow et al., 2004).

En relación a la elección de mazos en la tarea normal, los sujetos controles suelen aprender de forma implícita el funcionamiento de la prueba, ya que en el inicio tienden a seleccionar los mazos $B$ y $C$, pero a medida que avanzan se establece una preferencia por los mazos $C$ y $D$ (ambos favorables). Según la hipótesis del marcador somático, estos sujetos asignan diferentes valores emocionales a los mazos en función de sus experiencias previas de recompensa y castigo con los mismos. Este aprendizaje les permite generar respuestas emocionales que anticipan las consecuencias que se derivan de seleccionar cartas de los mazos desfavorables, guiando sus elecciones hacia los mazos favorables. Sin embargo, otros autores consideran que para ejecutar con éxito la IGT el sujeto debe tener la capacidad de establecer relaciones estímulo-recompensa, así como la habilidad para invertir o extinguir respuestas previamente aprendidas (Maia y McClelland, 2004; Rolls, 2004).

En los consumidores de cannabis no se produce la misma tendencia que en los controles, ya que tienden a seleccionar con mayor frecuencia los mazos B (desfavorable) y D (favorable) tanto en la fase inicial como final de la tarea, sin producirse un cambio de tendencia. Esto, según la hipótesis del marcador somático puede deberse a una incapacidad para utilizar señales emocionales en el momento de valorar diferentes opciones de respuesta (Bechara y Damasio, 2002; Damasio, 1994/2004), o bien, a la incapacidad para evaluar los efectos positivos o negativos de los actos, a la hipersensibilidad hacia las gratificaciones inmediatas, a la insensibilidad a los castigos, a la preferencia de opciones arriesgadas, a déficit en la memoria de trabajo o a la impulsividad. Otras hipótesis (Maia y McClelland, 2004; Rolls, 2004) sugieren que puede deberse a problemas en la inversión de aprendizajes previos, es decir, que los consumidores de cannabis presenten dificultades para modificar o extinguir respuestas a contingencias ambientales que previamente eran recompensadas.

Tanto en los consumidores de cannabis como en el grupo control existen diferencias estadísticamente significativas entre las elecciones del mazo $A$ y $B$, seleccionando con mayor frecuencia el mazo $B$, lo que indica que no es percibido como desfavorable. Varios autores (Lin et al., 2009; Lin et al., 2007) han intentado explicar este fenómeno, determinando que la preferencia de los mazos puede estar asociada a la frecuen- 
cia de ganancias y pérdidas, y no a los resultados obtenidos a largo plazo.

Respecto a la influencia de las variables analizadas, los resultados sugieren que el rendimiento de ambos grupos en la tarea normal no se ve afectado por la edad y el sexo. El nivel de estudios si incide en los controles pero no en los consumidores de cannabis. La cantidad consumida a diario (número de cigarros) tampoco incide, tal y como se ha venido afirmando en otros estudios (Bechara et al., 2001; Verdejo et al., 2004). En cuanto a la edad de inicio al consumo nuestros datos muestran que no influye en la toma de decisiones, lo que no coincide con trabajos previos (Gruber et al., 2009; Pope et al., 2003; Wilson et al., 2000), si bien esto puede estar motivado por los datos de nuestros sujetos, ya que de los 41 fumadores de cannabis, tan sólo 11 presentan una edad de inicio superior a los 17 años.

Diversos estudios (Bolla et al., 2005; Fridberg et al., 2010; Mogedas y Alameda, 2011; Vélez et al., 2010; Verdejo-García et al., 2007; Whitlow et al., 2004) señalan que los resultados obtenidos por los consumidores de cannabis y otras drogas, pueden deberse a tres factores, bien porque son insensibles a las pérdidas, teniendo en consideración sólo las ganancias; bien porque son hipersensibles a las ganancias e ignoran las pérdidas; o bien son sensibles a las pérdidas y ganancias, pero ninguna de ellas es capaz de establecer la guia de las elecciones para extraer la información adecuada que permita predecir los resultados futuros, y por ello, prefieren aquellas cartas con mayor recompensa a corto plazo.

Para analizar estas posibilidades se utiliza una versión invertida, es decir, se cambian las pérdidas por ganancias y las ganancias por pérdidas, en este caso las pérdidas son regulares y las ganancias esporádicas. Según informa Damasio (1994/2004), con este tipo de tareas los sujetos lesionados frontales tiene un comportamiento similar al de los sujetos normales-sanos, por lo que la insensibilidad a las pérdidas no puede ser la explicación correcta. Como hemos podido apreciar en nuestros resultados, el IGi de los consumidores de cannabis es superior al de los controles, y también superior a su desempeño en la tarea normal. Las diferencias con los sujetos controles se ciñen a la parte inicial de la prueba, y además en el estudio por bloques podemos apreciar que salvo en el IG40 las diferencias entre ambos grupos no son significativas, por lo que podríamos afirmar que no hay diferencias entre la ejecución de los fumadores de cannabis y los controles en la tarea inversa. Con estos datos también podemos apoyar la idea de que los malos resultados de los distintos IG en la prueba normal no se deben a la insensibilización a las pérdidas. En cuanto a las estrategias de elecciones de mazos, son similares a las empleadas en la prueba normal. Estos resultados coinciden con los de otros trabajos previos (Mogedas y Alameda, 2011), y hasta cierto punto también coinciden con los resultados informados por Damasio (1994/2004), quedando patente una mejor ejecución de los fumadores de cannabis en esta modalidad inversa que en la versión normal, si bien las diferencias entre ambas versiones se ciñe a las fases iniciales de la tarea.

En resumen, los resultados obtenidos indican que los consumidores de cannabis presentan dificultades en la toma de decisiones, evaluada a través del programa "Cartas", sin embargo, el rendimiento en dicha tarea no se ve influenciado por la edad, sexo, nivel de estudios, cantidad de cigarros consumidos a diario, ni por la edad de inicio del consumo. Los consumidores de cannabis, en comparación a los sujetos controles, están más influenciados por la búsqueda de la recompensa inmediata, presentan una menor aversión a las pérdidas y están más influenciados por los resultados recientes (Fridberg et al., 2010), aspectos que pueden estar relacionados con el funcionamiento anormal de las regiones prefrontales, especialmente la corteza orbitofrontal (Bechara, 2003; Fridberg et al., 2010; Verdejo-García et al., 2007; Withlow et al., 2004), ya que pueden estar integrando de forma deficiente la información relacionada con la recompensa sin tener en cuenta la retroalimentación negativa. Varios estudios (Bechara y Damasio, 2002; Verdejo et al., 2004) indican que este deterioro en la toma de decisiones podría deberse a una alteración o vulnerabilidad previa al inicio del consumo, o al efecto dicotómico (todo o nada) de la neurotoxicidad de las drogas.

Teniendo en cuenta lo dicho anteriormente, consideramos necesario abordar en futuras investigaciones otras cuestiones, como analizar el tiempo de consumo, incrementar la muestra para poder analizar mejor el papel de la edad de inicio usando grupos extremos en la misma, y controlar los períodos de abstinencia y número de recaídas. Además, siguiendo a Contreras, Catena, Cándido, Perales y Maldonado (2008), sería necesario buscar medidas más fiables y solucionar los problemas de previsibilidad, la programación de los mazos, especialmente en B y C (Lin et al., 2009; Lin et al., 2007; Mogedas y Alameda, 2011; van den Bos, Houx y Spruijt, 2006), buscar presentaciones de estímulos más intuitivas o informativas (Gordillo et al., 2010), o analizar el tipo de instrucciones (Balodis et al., 2006; DeDonno y Demaree, 2008; Fernie y Tunney, 2006).

\section{Conflicto de intereses}

No hay conflicto de intereses.

\section{Referencias}

Balodis, I.M., MacDonald, T.K. y Olmstead, M.C. (2006). Instructional cues modify performance on the lowa Gambling Task. Brain and Cognition, 60, 109-117.

Bechara, A. (2003). Risky business: Emotion, decision making, and addiction. Journal of Gambling Studies, 19, 102-103.

Bechara, A. y Damasio, H. (2002). Decision-making and addiction (par. I): Impaired activation of somatic states in substance dependent individuals when pondering decisions with negative future consequences. Neuropsychologia, 40, 1675-1689.

Bechara A., Damasio H. y Damasio, A.R. (2000). Emotion, decisiónmaking and the orbitofrontal cortex. Cerebral Cortex, 10, 295-307.

Bechara, A., Damasio, H., Damasio, A.R. y Anderson, S.W. (1994). Insensitivity to future consequences following damage to human prefrontal cortex. Cognition, 50, 7-15. 
Bechara, A., Dolan, S., Denburg, N., Hindes, A., Anderson, S.W. y Nathan, P.E. (2001). Decision-making deficits, linked to a dysfunctional ventromedial prefrontal cortex, revealed in alcohol and stimulant abusers. Neuropsychologia, 39, 376-389.

Bolla, K.I., Brown, K., Eldreth, D., Tate, K. y Cadet, J.L. (2002). Doserelated neurocognitive effects of marijuana use. Neurology, 59, 1337-1343.

Bolla, K.I., Eldreth, D.A., Matochik, J.A. y Cadet, J.L. (2005). Neural substrates of faulty decision-making in abstinent marijuana users. Neuroimage, 29, 480-492. Doi: 10.1016/j.neuroimage.2005.02.012

Bos, R. van den, Houx, B.B. y Spruijt, B.M. (2006). The effect of reward magnitude differences on choosing disadvantageous decks in the lowa Gambling Task. Biologycal psichology, 71, 155-161.

Chang, L., Yakupov, R., Cloak, C. y Ernst, T. (2006). Marijuana use is associated with a reorganized viaula-attention network and cerebellar hypoactivation. Brain, 129, 1096-1112. Doi: 10.1093/ brain/awl064

Clark, L., Cools, R. y Robbins, T.W. (2004). The neuropsychology of ventral prefrontal cortex: Decision making and reversal learning. Brain and Cognition, 55, 21-53.

Contreras, D., Catena, A., Cándido, A., Perales, J.C. y Maldonado, A. (2008). Funciones de la corteza prefrontal ventromedial en la toma de decisiones emocionales. Internacional Journal of Clinical and Health Psychology, 8(1), 285-313.

Damasio, A. (2004). El error de Descartes. Barcelona: Crítica. (Orig. 1994).

DeDonno, M.A. y Demaree, H.A. (2008). Perceived time pressure and de lowa Gambling Task. Judgment and Decision Making, 3, 636-640.

Delegación del Gobierno para el Plan Nacional sobre Drogas. Observatorio Español sobre Drogas (OED). Informe 2009. Situación y tendencias de los problemas de drogas en España. Madrid: Ministerio de Sanidad y Política Social.

Dunn, B.D., Dalgleish, T. y Lawrence, A.D. (2006). The somatic marker hypothesis: A critical evaluation. Neuroscience and Biobehavioral reviews, 30, 239-71.

European Monitoring Center for Drugs and Drug Addiction (2010). Informe Anual 2010: El problema de la drogodependencia en Europa. Luxemburgo: Oficina de publicaciones de la Unión Europea.

European Monitoring Center for Drugs and Drug Addiction (2008). A cannabis reader: global sigues and local experiences. Luxemburgo: Office for Official Publications of the European Communities.

Facundo, A.B., Martín-Santos, R., Abades, S., Farré, M. y Verdejo-Garcia, A. (2008). Neuroimagen y adicción II: Correlatos neuroanatómicos y funcionales de la administración aguda, el craving y el consumo crónico de opiáceos, alcohol y cannabis. Revista Española de Drogodependencias, 32, 125-149.

Fernie, G. y Tunney, R.J. (2006). Some decks are better than others: The effect of reinforcer type and task instructions on learning in the lowa Gambling Task. Brain and Cognition, 60, 94-102.

Fridberg, D.J., Queller, S., Ahn, W., Kim, W., Bishara, A., Busemeyer, J.R.,...Stout, J.C. (2010). Cognitive mechanisms underlying risky decision-making in chronic cannabis users. Journal of Mathematical Psychology, 54, 28-38. Doi: 10.1016/j.jmp.2009.10.002

Fried, P.A., Watkinson, B. y Gray, R. (2005). Neurocognitive consequences of marihuana comparison with pre-drug performance. Neurotoxicology and Teratology, 27, 231-239.

Grant, J.E., Chamberlain, S.R., Schreiber, L. y Odlaung, B.L. (2011). Neuropsychological deficits associated with cannabis use in young adults. Drug and Alcohol Dependence. Avance de publicación on-line. Doi: 10.1016/j.drugalcdep.2011.08.015
Grant, S., Conttoreggi, C. y London, E.D. (2000). Drug abusers show impaired performance in a laboratoy test of decision-making. Neuropsychologia, 38, 1180-1187.

Gruber, S.A., Rogowska, J. y Yurgelun-Todd, D. (2009). Altered Affective Response in Marijuana Smokers: An FMRI Study. Drug and Alcohol Dependence, 105, 139-153. Doi: 10.1016/j.drugalcdep.2009.06.019

Hall, W. y Degenhardt, L. (2009). Adverse health effects of nonmedical cannabis use. The Lancet, 374, 1383-1391.

Jager, G., Kahn, R.S., van den Brink, W., van Ree, R.M. y Ramsey, N.F. (2006). Long-term effects of frequent cannabis use on working memory an attention: an fMRI study. Psychopharmacology, 185, 358-368.

Kelleher, L. M., Stough, C., Sergejew, A.A. y Rolfe, T. (2004). The effects of cannabis on information-pricessing speed. Addictive Behaviors, 29, 1213-1219.

Lin, C.H., Chiu, Y.C. y Huang, J.T. (2009). Gain-loss frequency and final outcome in the Soochow Gambling Task: A Reassessmente. Behavioral and Brain Function, 5:45.

Lin C.H., Chiu Y.C., Lee P.L. y Hsieh J.C. (2007). Is deck B a disadvantageous deck in the lowa Gambling Task?". Behavioral and Brain Function, 3:16. Doi: 10.1186/1744-9081-3-16

Lundqvist, T. (2005). Cognitive consequences of cannabis use: comparison with abuse of stimulants and heroin with regard to attention, memory and executive functions. Pharmacology Biochemistry and Behavior, 81, 319-330. Doi: 10.1016/j. pbb.2005.02.017

Maia, T.V. y McClelland, J.L. (2004). A reexamination of the evidence for the somatic marker hypothesis: what participants really know in the lowa gambling task. Proceedings of National Academy of Sciences of the United States of America, 101, 16075-16080.

Mazas, C., Finn, P., y Steinmetz, J.E. (2000). Decision-Making biases, antisocial personality, and Early-Onset Alcoholism. Alcoholism: Clinical and Experimental Research, 24, 106-140.

Mogedas, A.I. y Alameda, J.R. (2011). Toma de decisiones en pacientes drogodependientes. Adicciones, 23, 277-287.

Palacios, E., Paino, S.G. y Alameda, J.R. (2010). Programa Cartas. Descargado: http://www.telecable.es/personales/elias.palacios/ CartasSetup.jar.

Pillay, S.S., Rogowska, J., Kanayama, F., Jon, D.I., Gruber, S., Simpson, N., ... Yurgelun-Todd, D. (2004). Neurophysiology of motor function following cannabis discontinuation in chronic cannabis smokers: an fMRI study. Drug and Alcohol Dependence, 76, 261-271.

Pope, J., Gruber, A. J., Hundon, J.L., Cohane, G., Huestes, M.A. y Yurgelun-Todd, D. (2003). Early-onset cannabis use and cognitive deficits: what is the neture of the association? Drug and Alcohol Dependence, 69, 303-310.

Pope, H.G., Gruber, A.J., Hudson, J.L., Huestis, M.A. y Yurgelun-Todd, D. (2001). Neuropsychological performance in long-term cannabis users. Archives of General Psychiatry, 58, 909-915.

Rolls, E.T. (2004). The functions of the orbitofrontal cortex. Brain and Cognition, 55, 11-29.

Schwartz, R.H., Gruenewald, P.J., Klitzner, M. y Fedio, P. (1986). Shortterm memory impairment in cannabis-dependent adolescents. American Journal of Diseases in Childhood, 143, 1214-1219.

Shannon, E.E., Mathias, C.W., Dougherty, D.M. y Anthony Liguori, A. (2010). Cognitive Impairments in Adolescent Cannabis Users are Related to THC Levels. Addictive Disorders \& Their Treatment, 9, 158-163. 
Slowij, N. y Grenyer, B.F.S. (2002). Are the adverse consequences of cannabis use age-dependent. Addiction, 97, 1083-1086. Doi: 10.1046/j.1360-0443.2002.00243.x

Vélez, A., Borja, K. y Ostrosky-Solís, F. (2010). Efectos del consumo de marihuana sobre la toma de decisión. Revista Mexicana de Psicología, 27, 309-315.

Verdejo, A., Aguilar, F. y Pérez-García, M. (2004). Alteraciones de los procesos de toma de decisiones vinculados al córtex prefrontal ventromedial en pacientes drogodependientes. Revista de Neurología, 38, 601-606.

Verdejo, A., López-Torrecillas, F., Orozco, C. y Pérez, M. (2002). Impacto de los deterioros neuropsicológicos asociados al consumo de sustancias sobre la práctica clinica en drogodependientes. Adicciones, 14, 345-370.

Verdejo-Garcia, A., Benbrook, A., Funderburk, F., David, P., Cadet, J.L. y Bolla, K.I. (2007). The diferential relationship between cocaine use and marijuana use on decision-making performance over repeat testing with the lowa Gambling Task. Drug and Alcohol Dependence, 90, 2-11

Verdejo-García, A., Pérez-Garcia, M., Sánchez-Barrera, M., RodríguezFernández, A. y Gómez-Río, M. (2007). Neuroimagen y drogodependencias: correlatos neuroanatómicos del consumo de cocaína, opiáceos, cannabis y éxtasis. Revista de neurología, 44, 432-439.

Whitlow, C.T., Liguari, A., Livengook, L.B., Hart, S.L., Mussat-Whitlow, B.J., Lamborn, C.M., ... Porrino, L.J. (2004). Long-term heavy marijuana users make costly decisions on a gambling task. Drug and alcohol Dependence, 76, 107-111.

Wilson, W., Mathew, R., Turkington, T., Hawk, T., Coleman, R.E. y Provenzale, J. (2000). Brain morphological changes and early marijuana use: a magnetic resonance and positron emission tomography study. Journal of Addictive Disease, 19, 1-22. 